\title{
Perceived well-being in adolescent immigrants: it matters where they come from
}

\author{
Alberto Borraccino ${ }^{1} \cdot$ Lorena Charrier $^{1} \cdot$ Paola Berchialla $^{2} \cdot$ Giacomo Lazzeri $^{3} \cdot$ Alessio Vieno $^{4}$. \\ Paola Dalmasso ${ }^{1} \cdot$ Patrizia Lemma $^{1}$
}

Received: 6 June 2018/Revised: 4 September 2018 / Accepted: 1 October 2018

(C) Swiss School of Public Health (SSPH+) 2018

\begin{abstract}
Objectives The aim of this work was to explore whether adolescent immigrants have worse or better perceived well-being, and whether this perception varies by ethnic background or between first- and second-generation immigrants, when compared with adolescents from the host population.

Methods A representative sample of 47,799 students (13.4\% immigrants) aged 11, 13, and 15 years were recruited throughout all Italian regions within the framework of the Italian 2013/2014 Health Behaviour in School-aged Children study.

Results Adolescent immigrants from Eastern European and non-Western/non-European countries had a higher occurrence of health complaints and the highest risk of reporting low life satisfaction, which increased among the second generation. The risk of reporting bullying behaviours and physical fights was higher in first-generation immigrants and decreased in the second generation, independent of ethnic background.

Conclusions Italian welfare immigration policies do not seem to offer the same opportunities to all adolescent immigrant groups, which leads to differing effects on their well-being. To tackle these inequalities, Italy should increase its investment in education through early prevention initiatives, e.g. providing support in intercultural education and proper training to school teachers and staff.
\end{abstract}

Keywords First and second adolescent immigrants' generation · Perceived well-being · Bullying behaviours . Physical fights · HBSC

\section{Introduction}

Electronic supplementary material The online version of this article (https://doi.org/10.1007/s00038-018-1165-8) contains supplementary material, which is available to authorized users.

\footnotetext{
Alberto Borraccino

alberto.borraccino@unito.it

Lorena Charrier

lorena.charrier@unito.it

Paola Berchialla

paola.berchialla@unito.it

Giacomo Lazzeri

giacomo.lazzeri@unisi.it

Alessio Vieno

alessio.vieno@unipd.it

Paola Dalmasso

paola.dalmasso@unito.it
}

Immigration has increased in the last years in most Western European countries, and one of the main related aspects that deserves more attention is the health of children and

Patrizia Lemma

patrizia.lemma@unito.it

1 Department of Public Health and Paediatrics, University of Torino, Piazza Polonia, 94, 10126 Turin, Italy

2 Department of Clinical and Biological Sciences, University of Torino, Turin, Italy

3 Department of Molecular and Developmental Medicine, University of Siena, Siena, Italy

4 Department of Developmental and Social Psychology, University of Padova, Padua, Italy 
adolescents with an immigrant background, as they did not choose to leave their home country, and they suffer the same poverty and discrimination as their families upon arrival in the host country (Mood et al. 2016). Moreover, in countries with low birth rates, adolescent immigrants constitute a sizeable, expanding proportion of the population (Hernandez 2010; Sam et al. 2008). Just like their native-born counterparts, immigrant youths have to develop skills and relationships both in school (academic achievements, and relationships with teachers and peers) and in their family for their own growth and psychological well-being (Stefanek et al. 2012). However, immigrant youths are faced with the additional challenge of their 'acculturation process' (Resnick et al. 1997), which can have different outcomes: integration, assimilation, separation, or marginalisation (Berry and Sabatier 2011; Berry and Sam 1997).

Well-being represents a balance, by which 'individuals have the psychological, social, and physical resources they need to meet a particular challenge' (Dodge et al. 2012). Without this balance, a person's perceived well-being may worsen; this can be experienced as internalising problems (including depression, anxiety, sleep disorders, or withdrawal symptoms) and externalising behaviours (including aggression, oppositional disorders, or delinquency) (Kim et al. 2003). Critical stressful life events, such as economic or familial difficulties, including immigration itself (Stefanek et al. 2012), particularly at developmental ages, seem to predict changes in both internalising problems and externalising behaviours (Kim et al. 2003; Kwak 2016). In order to reduce the risk of marginalisation, one of the biggest challenges that host countries face with respect to young immigrants is early detection of worsening wellbeing (Kouider et al. 2015).

Immigrant status in itself has often been postulated as a risk factor for mental health problems among children and adolescents, particularly in first-generation immigrants, but previous empirical findings have pointed in opposite directions (Harker 2001; Kouider et al. 2014, 2015; Mood et al. 2016; Stevens et al. 2015). A longitudinal study from the USA showed that first-generation immigrants appear to have a higher level of positive well-being and a lower level of depression than their native counterparts (Harker 2001). Conversely, a recent systematic review on native and immigrant children in American countries showed balanced results in regard to mental disorders, with a slight tendency of more internalising problems among immigrants with an Asian ethnic background (Kouider et al. 2015).

European studies have also reported contrasting results. In their review of immigration in Europe, Kouider et al. (2014) reported an increased risk of internalising problems; but the presence of externalising behaviours was comparable between native-born and immigrant populations. However, that review did not take into account any differences between first- and second-generation immigrants. Stevens et al. (2015) compared nine European countries and the USA and found a very weak first-generation disadvantage, but no second-generation disadvantage in psychosomatic symptoms and life satisfaction, and an overall disadvantage in bullying behaviours and physical fights. In their wide longitudinal survey conducted in England, Germany, Netherlands, and Sweden, Mood et al. (2016) reported fewer internal and external problems in young immigrants than in the host population, further supporting the 'immigrant health paradox' found in some North American (Harker 2001; Kouider et al. 2015) and European studies. To date, there has been a conspicuous lack of systematic patterns in the literature. Most of all, there have been few studies that compared well-being in more than one generation of adolescent immigrants (Kwak 2016).

Italy has recently experienced large waves of immigration, and the number of first- and second-generation immigrants is growing each year (ISTAT 2016). There were 800,000 adolescents with an immigrant background in Italy in 2014, representing nearly $14 \%$ of the population in compulsory schools (Dalmasso et al. 2017; MIUR 2015), and there is a specific interest in exploring differences in well-being between adolescent immigrants and their native-born counterparts. Therefore, this study aimed to explore whether adolescent immigrants have worse or better perceived well-being, and whether this perception varies by ethnic background or between first- and secondgeneration immigrants, when compared with adolescents from the host population.

\section{Methods}

The Italian Health Behaviour in School-aged Children (HBSC) study protocol and questionnaire were formally approved on 21 March 2012 by the Bioethics Committee of the University of Torino. Consent was obtained from local authorities, school administrators, parent/guardians, and students. Parents and students were able to opt out of any questionnaire item. No reimbursement was planned nor provided for participation.

\section{Study population and design}

Data were collected as part of the Italian 2013/2014 HBSC study. The HBSC study is a World Health Organisation (WHO) Collaborative Cross-National Survey, which runs every 4 years. In 2014, it included 43 countries across and outside Europe. To ensure consistency in the survey 
instruments, informed consent procedures, anonymity and confidentiality, and data collection and processing, each country adheres to the HBSC international study protocol (Currie et al. 2014). School is the primary sampling unit, drawn by systematic cluster sampling from a list of all public and private schools. In Italy, this list was obtained from the Italian Ministry of Education, University and Research (MIUR), and a representative sample of students aged 11, 13, and 15 years from 3681 school classes located throughout all Italian regions were invited to participate. Of these, 3315 classes agreed (response rate: $90.1 \%$ ), and a final sample of 47,799 students were enrolled. The study questionnaires were administered by ad hoc trained personnel and collected information on ethnic background, socioeconomic status (SES), and well-being. Four hundred students with missing data on ethnic background (1\%) were excluded. Results are based on a total of 47,399 valid questionnaires.

\section{Variables and measures}

Adolescents were classified as being from the 'host population' if both parents were born in Italy, independently of the child's country of birth; as 'first-generation immigrants' if they were born abroad and at least one of their parents was born abroad; and as 'second-generation immigrants' if they were born in Italy and at least one of their parents was born abroad (Currie et al. 2014).

Ethnic background was defined as the country of birth of the student's mother. However, if the mother's country of birth was missing or she was born in Italy, ethnic background was defined as the country of birth of the student's father. Based on this information, adolescent immigrants' ethnic backgrounds were categorised as follows (Dalmasso et al. 2017):

- Western countries $(n=2195)$ - This category included European Union (EU)-15 countries (EU member states prior to May 2004), Switzerland, Norway, and Iceland. This category also included the USA, Canada, Australia, and New Zealand, all of which are classified by the International Monetary Fund as countries with advanced economies (Eurostat 2018);

- Eastern European countries $(n=2424)$-This category included EU-13 countries (new member states that joined the EU after May 2004), Albania, Bosnia, Macedonia, Moldavia, Serbia, and Ukraine (Eurostat 2018);

- Non-Western/non-European countries $(n=2556)$ This category included Africa, South or Central America, and Asia.

SES was measured according to the family affluence scale (FAS), which has been recognised as a reliable indicator of family wealth (Torsheim et al. 2016). The scale consists of six items: family car ownership, whether adolescents have their own bedroom, number of holidays trips taken in the last year, number of computers owned by the family, dishwasher ownership, and number of bathrooms in the home. The obtained score $(0-13)$ was categorised as low (0-6), medium (7-9), and high $(\geq 10)$ SES (Currie et al. 2014; Torsheim et al. 2016).

\section{Outcome measures}

Well-being was measured as internalising problems (health complaints and life satisfaction) and externalising behaviours (bullying behaviours and physical fights). Health complaints were assessed through an eight-item checklist (Haugland and Wold 2001) that recorded how often participants had experienced the following symptoms in the past 6 months: stomach ache, headache, backache, feeling low, irritability or bad temper, feeling nervous, difficulties in getting to sleep, and feeling dizzy. Responses were given on a five-point scale: rarely or never (0); about every month (1); about every week (2); more than once a week (3); and about every day (4). A summary scale was created with a range of $0-32$ points, with a higher score indicating a higher frequency of health complaints (Ravens-Sieberer 2004). In the analyses, health complaints were treated as a continuous variable and reported as means.

Life satisfaction was assessed with the Cantril ladder (Cantril 1965). Respondents were asked to indicate where they would say their position on the ladder was at that moment, with the bottom of the ladder (0) representing the worst way of living and the top (10) indicating the best possible way of living. Findings were dichotomised as low (0-5) and normal-to-high life satisfaction (6-10) (Currie et al. 2010).

Bullying behaviours were measured by asking how often participants had taken part in bullying another student at school. Responses were dichotomised into none and at least once in the past couple of months (Pickett et al. 2009). Physical fights were measured by asking students how many times in the past 12 months they had been involved in a fight in school. Responses were dichotomised into never and one or more times (Djerboua et al. 2016).

\section{Statistical analysis}

Descriptive analyses were used to compare the distribution of first-generation immigrants, second-generation immigrants, and the host population by sex, age, and SES (FAS category). The corrected, weighted Pearson Chi-square statistic was used to compare the occurrence of internalising problems (health complaints and life satisfaction) and 
externalising behaviours (bullying behaviours and physical fights) among the subgroups in the analysis.

Several multivariable linear and logistic regression models were used to fit the association between ethnic background as the independent variable and health complaints, life satisfaction, bullying behaviours, and physical fights as dependent variables, using the host population as the reference category. All models were run within each socio-demographic variable (age, sex, SES) and mutually adjusted for the others.

The influence of being a first- or second-generation immigrant was evaluated using the same multivariable linear and logistic regression models after combining Eastern European countries and non-Western/non-European countries into one category, as they have been shown to have a comparable profile (Dalmasso et al. 2017), see supplementary material. All analyses were performed while taking into consideration the effect of the survey design; thus, they included stratification, clustering, and weighting. All analyses were performed in STATA v14.1 (StataCorp., College Station, TX, USA), and a statistical significance level of $5 \%$ was used.

\section{Results}

First- or second-generation immigrants represented $13.4 \%$ of the entire study sample $(4.0 \%$ from Western countries, $4.1 \%$ from Eastern European countries, and 5.3\% from non-Western/non-European countries), with the remaining $86.6 \%$ coming from the host population. Adolescent immigrants from Eastern European and non-Western/nonEuropean countries were more likely to have low SES ( $43.7 \%$ vs $46.8 \%$ respectively) than the host population (low $\mathrm{SES}=33 \%$ ), whereas immigrants from Western countries had a higher SES in both generations $(p<0.0001)$ (Table 1).

When looking at internalising problems, health complaints were more common in immigrant adolescents from Eastern European countries, with statistically significant

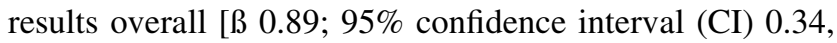
1.43 ], by sex [boys: $\beta 0.78 ; 95 \%$ CI 0.04 , 1.51 , girls: $\beta$ $0.93 ; 95 \%$ CI $0.17,1.69]$, and by age [15-year-olds: $\beta 1.47$; 95\% CI 0.34, 2.61]. Immigrant adolescents from Eastern European countries reported a mean amount of symptoms that was more than one symptom higher than that of the host population. The likelihood of reporting low life satisfaction was significantly higher among adolescent immigrants from Eastern European and non-Western/nonEuropean countries, in both sexes and in all investigated age groups (Table 2).

As for externalising behaviours, adolescent immigrants from non-Western/non-European countries reported a significantly higher odds ratio (OR) for bullying behaviours than their counterparts from the host population [OR 1.33; $95 \%$ CI 1.10, 1.62], the same pattern was also observed among girls [OR 1.48; 95\% CI 1.11, 1.96]. Physical fights were reported more frequently by adolescent immigrants from Eastern European and non-Western/non-European countries [OR 1.29; 95\% CI 1.07, 1.55 and 1.36; 95\% CI 1.17 , 1.58, respectively], with a higher, significant risk among Eastern European girls [OR 1.37; 95\% CI 1.03, 1.82)] and among non-Western/non-European boys [OR $1.51 ; 95 \%$ CI 1.22, 1.86] (Table 2).

First-generation adolescent immigrants from Western countries reported fewer health complaints than their counterparts from the host country (Table 3). However, adolescent immigrants from Eastern European and nonWestern/non-European countries showed a higher occurrence of health complaints in both generations, although this difference was only statistically significant in the second generation [mean 11.2, ß 0.70; 95\% CI 0.25, 1.15].

With regard to life satisfaction, the profile of adolescent immigrants from Western countries showed overlapped with that of adolescents from the host country. On the other hand, both first- and second-generation immigrants from Eastern and non-Western/non-European countries were more likely to report low life satisfaction [OR 1.59; $95 \%$ CI 1.39, 1.80 and OR 1.65; 95\% CI 1.50, 1.81, respectively].

The likelihood of bullying behaviours was high among both generations of adolescent immigrants from Western countries [OR 1.68; 95\% CI 1.30, 2.08] and those from Eastern European and non-Western/non-European countries [OR 1.62; 95\% CI 1.39, 1.85], although results were statistically significant only for the first generation. Physical fights were more frequent among immigrants from Eastern and non-Western/non-European countries, with a significant OR of 1.41 [95\% CI 1.25, 1.57] among the first generation and of 1.23 [95\% CI 1.12, 1.34] among the second, when compared with their host country counterparts. Adolescent immigrants from Western countries showed a profile that was similar to that of the host population (Table 3).

\section{Discussion}

In the present study, we used self-reported assessments of internalising problems (health complaints and life satisfaction) and externalising behaviours (bullying behaviours and physical fights) to measure perceived well-being among first-generation and second-generation adolescent immigrants, and the host population in Italy. We then compared the information from adolescent immigrants to information from the host population. The goal was to 


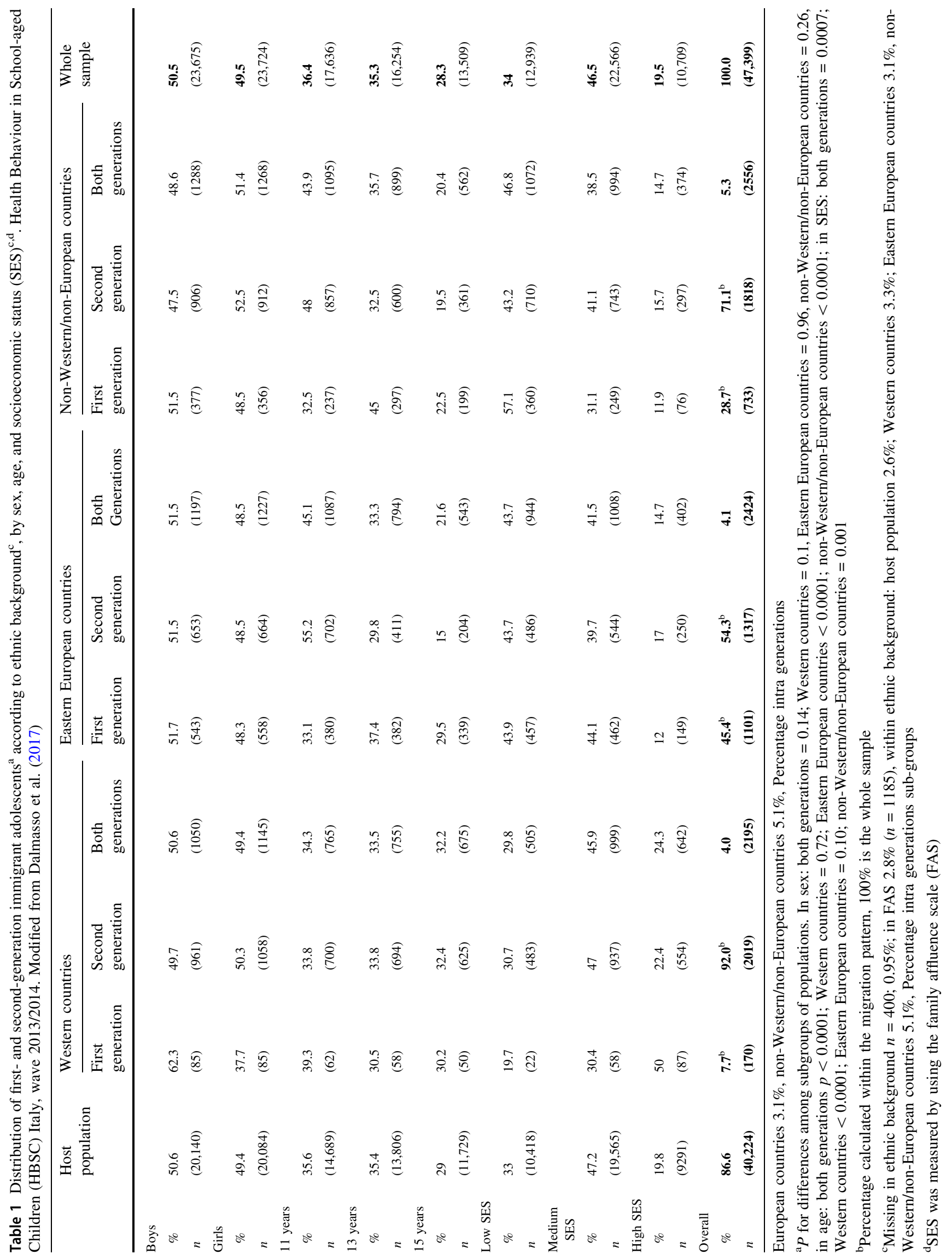


Table 2 Distribution, mean, $\beta$ score, and $\mathrm{OR}^{\mathrm{a}}$ of internalised problems (health complaints and low life satisfaction) and externalising behaviours (bullying behaviours and physical fights) according to ethnic background, sex, and age. Reference category is the host population. Health Behaviour in School-aged Children (HBSC) Italy, wave 2013/2014

\begin{tabular}{|c|c|c|c|c|c|c|c|c|c|}
\hline \multirow[t]{2}{*}{ Internalising problems } & \multicolumn{3}{|c|}{ Host population } & \multicolumn{2}{|c|}{ Western countries } & \multicolumn{2}{|c|}{ Eastern European countries } & \multicolumn{2}{|c|}{$\begin{array}{l}\text { Non-Western/non-European } \\
\text { countries }\end{array}$} \\
\hline & & Mean & $\beta$ & Mean & $\beta(95 \% \mathrm{CI})$ & Mean & $\beta(95 \% \mathrm{CI})$ & Mean & $\beta(95 \% \mathrm{CI})$ \\
\hline \multirow{7}{*}{$\begin{array}{l}\text { Health complaints } \\
\text { [range 0-32] }\end{array}$} & & 10.5 & Ref. & 10.5 & $0.02(-0.52,0.55)$ & 11.4 & $0.89(0.34,1.43)^{* *}$ & 10.9 & $0.44(-0.04,0.92)$ \\
\hline & Boys & 8.7 & Ref. & 8.8 & $0.11(-0.59,0.81)$ & 9.4 & $0.78(0.04,1.51)^{* *}$ & 9.2 & $0.57(-0.14,1.28)$ \\
\hline & Girls & 12.3 & Ref. & 12.2 & $-0.15(-0.93,0.64)$ & 13.2 & $0.93(0.17,1.69)^{* *}$ & 12.7 & $0.39(-0.29,1.07)$ \\
\hline & 11 years & 9.1 & Ref. & 9.1 & $-0.04(-0.86,0.79)$ & 9.7 & $0.55(-0.20,1.30)$ & 9.8 & $0.70(-0.03,1.43)$ \\
\hline & 13 years & 10.8 & Ref. & 11.1 & $0.31(-0.58,1.19)$ & 11.7 & $0.83(-0.18,1.84)$ & 10.8 & $-0.04(-0.84,0.77)$ \\
\hline & 15 years & 11.8 & Ref. & 11.4 & $-0.35(-1.36,0.66)$ & 13.3 & $1.47(0.34,2.61)^{* *}$ & 12.7 & $0.89(-0.14,1.92)$ \\
\hline & & $\%$ & OR & $\%$ & OR $(95 \% \mathrm{CI})$ & $\%$ & OR $(95 \% \mathrm{CI})$ & $\%$ & OR $(95 \% \mathrm{CI})$ \\
\hline Low life satisfaction & All & 14.4 & Ref. & 15.4 & $1.08(0.86-1.36)$ & 21.8 & $1.73(1.43-2.08)^{* *}$ & 21.0 & $1.61(1.35-1.93)^{* *}$ \\
\hline & Boys & 10.8 & Ref. & 11.8 & $1.08(0.77-1.52)$ & 14.6 & $1.46(1.08-1.97)^{* *}$ & 18.0 & $1.74(1.32-2.30)^{* *}$ \\
\hline & Girls & 18.1 & Ref. & 19.1 & $1.08(0.80-1.47)$ & 29.3 & $1.95(1.53-2.48)^{* *}$ & 23.9 & $1.53(1.20-1.94)^{* *}$ \\
\hline & 11 years & 11.3 & Ref. & 8.8 & $0.70(0.46-1.06)$ & 16.2 & $1.51(1.13-2.00)^{* *}$ & 16.8 & $1.46(1.07-1.98)^{* *}$ \\
\hline & 13 years & 14.9 & Ref. & 16.7 & $1.24(0.86-1.78)$ & 23.5 & $1.77(1.31-2.39)^{* *}$ & 22.1 & $1.61(1.19-2.17)^{* *}$ \\
\hline & 15 years & 17.5 & Ref. & 21.1 & $1.25(0.85-1.84)$ & 30.6 & $2.00(1.35-2.97)^{* *}$ & 28.3 & $1.85(1.35-2.55)^{* *}$ \\
\hline Externalising behaviours & & $\%$ & OR & $\%$ & OR $(95 \% \mathrm{CI})$ & $\%$ & OR $(95 \% \mathrm{CI})$ & $\%$ & OR $(95 \% \mathrm{CI})$ \\
\hline Bullying behaviours & All & 15.3 & Ref & 17.4 & $1.18(0.97-1.44)$ & 18.3 & $1.21(0.98-1.51)$ & 19.7 & $1.33(1.10-1.62)^{*}$ \\
\hline & Boys & 18.8 & Ref & 21.1 & $1.16(0.89-1.52)$ & 23.2 & $1.27(0.94-1.72)$ & 23.7 & $1.23(0.94-1.60)$ \\
\hline & Girls & 11.8 & Ref & 13.6 & $1.21(0.88-1.65)$ & 13.2 & $1.13(0.82-1.55)$ & 16.1 & $1.48(1.11-1.96)^{* *}$ \\
\hline & 11 yea & 14. & Ref & 14.7 & $1.04(0.75-1.44)$ & 15.0 & $0.98(0.69-1.38)$ & 18.2 & $1.30(0.93-1.80)$ \\
\hline & 13 yea & 17.8 & Ref & 23.9 & $1.45(1.06-1.98)^{* *}$ & 18.4 & $1.00(0.72-1.40)$ & 20.8 & $1.17(0.87-1.56)$ \\
\hline & 15 yea & 13.3 & Ref & 13.6 & $1.02(0.67-1.54)$ & 24.9 & $2.34(1.53-3.58)^{* *}$ & 21.0 & $1.77(1.19-2.64) * *$ \\
\hline Physical fights & All & 31.6 & Ref & 32.2 & $1.07(0.90-1.27)$ & 37.1 & $1.29(1.07-1.55)^{* *}$ & 37.6 & $1.36(1.17-1.58)^{* * *}$ \\
\hline & Boys & 45.3 & Ref & 47.2 & $1.10(0.87-1.39)$ & 50.6 & $1.23(1.00-1.52)$ & 55.8 & $1.51(1.22-1.86)^{* *}$ \\
\hline & Girls & 17.7 & Ref & 17.2 & $1.02(0.75-1.38)$ & 23.0 & $1.37(1.03-1.82)^{* *}$ & 20.9 & $1.19(0.93-1.52)$ \\
\hline & 11 yea & 34.0 & Ref & 38.5 & $1.23(0.93-1.62)$ & 35.0 & $1.02(0.77-1.35)$ & 37.6 & $1.27(1.00-1.61)$ \\
\hline & 13 yea & 33.7 & Ref & 35.2 & $1.04(0.78-1.38)$ & 41.9 & $1.45(1.04-2.01)^{* *}$ & 40.0 & $1.40(1.11-1.77)^{* *}$ \\
\hline & 15 yea & 26.1 & Ref & 22.6 & $0.93(0.65-1.33)$ & 33.9 & $1.74(1.22-2.48)^{* *}$ & 33.4 & $1.49(1.08-2.06)^{* *}$ \\
\hline
\end{tabular}

$O R$ odds ratio, $C I$ confidence interval, FAS family affluence scale

${ }^{a}$ All ORs are mutually adjusted for age, sex, and socioeconomic status, as measured by the FAS

$* * p<0.05$

highlight any differences that could be interpreted as early signs of psychosocial stress and used in early management efforts (Kim et al. 2003; Kwak 2016).

Based on the SES distribution among our defined ethnic backgrounds, our results identified two separate immigration patterns: a Western countries pattern and a second pattern that encompassed adolescent immigrants from Eastern European countries and non-Western/non-European countries. The differences between the pattern in Italian adolescents and the two observed immigration patterns were greatest in health complaints and life satisfaction. In fact, adolescent immigrants from Eastern European and non-Western/non-European countries reported low life satisfaction far more frequently than their counterparts from the host population, and the difference between these groups became more marked in the second generation. The same was true for the occurrence of health complaints: adolescent immigrants from Eastern European and nonWestern/non-European countries reported a higher occurrence than did the host population. This occurrence was statistically significant in second-generation adolescent immigrants from Eastern Europe. It was also statistically significant in both generations in adolescent immigrants from non-Western/non-European countries.

The picture that emerges from our results seems to be supported within the 'social stress' framework, which 
Table 3 Distribution, mean, $\beta$ score, and $\mathrm{OR}^{\mathrm{a}}$ of internalised problems (health complaints and low life satisfaction) and externalising behaviours (bullying behaviours and physical fights) according to ethnic background and immigrants' generation. Reference category is the host population. Health Behaviour in School-aged Children (HBSC) Italy, wave 2013/2014

\begin{tabular}{|c|c|c|c|c|c|c|c|c|c|c|}
\hline \multirow[t]{3}{*}{$\begin{array}{l}\text { Internalising } \\
\text { problems }\end{array}$} & \multirow{2}{*}{\multicolumn{2}{|c|}{$\begin{array}{l}\text { Host } \\
\text { population }\end{array}$}} & \multicolumn{4}{|c|}{ Western countries } & \multicolumn{4}{|c|}{$\begin{array}{l}\text { Eastern European countries and non-Western/non- } \\
\text { European countries }\end{array}$} \\
\hline & & & \multicolumn{2}{|c|}{ First generation } & \multicolumn{2}{|c|}{ Second generation } & \multicolumn{2}{|c|}{ First generation } & \multicolumn{2}{|c|}{ Second generation } \\
\hline & Mean & $\beta$ & Mean & $\beta(95 \% \mathrm{CI})$ & Mean & $\beta(95 \% \mathrm{CI})$ & Mean & $\beta(95 \% \mathrm{CI})$ & Mean & $\beta(95 \% \mathrm{CI})$ \\
\hline \multirow[t]{2}{*}{ Health complaints ${ }^{\mathrm{b}}$} & 10.5 & Ref. & 9.2 & $\begin{array}{l}-1.33(-1.9 \\
0.2)\end{array}$ & 10.6 & $\begin{array}{l}0.11(-0.5 \\
0.7)\end{array}$ & 10.9 & $\begin{array}{l}0.47(-0.07 \\
1.01)\end{array}$ & 11.2 & $\begin{array}{l}0.70(0.25 \\
1.15)^{* *}\end{array}$ \\
\hline & $\%$ & OR & $\%$ & OR $(95 \% \mathrm{CI})$ & $\%$ & OR $(95 \% \mathrm{CI})$ & $\%$ & OR $(95 \% \mathrm{CI})$ & $\%$ & OR $(95 \% \mathrm{CI})$ \\
\hline $\begin{array}{l}\text { Low life } \\
\text { satisfaction }^{\mathrm{b}}\end{array}$ & 14.4 & Ref. & 14.9 & $1.05(0.77-1.32)$ & 15.6 & $\begin{array}{l}1.09 \\
(0.91-1.26)\end{array}$ & 22.2 & $\begin{array}{l}1.59 \\
(1.39-1.80)^{* *}\end{array}$ & 20.9 & $\begin{array}{l}1.65 \\
(1.50-1.81)^{* *}\end{array}$ \\
\hline $\begin{array}{l}\text { Externalising } \\
\text { behaviours }\end{array}$ & $\%$ & OR & $\%$ & OR $(95 \% \mathrm{CI})$ & $\%$ & OR $(95 \% \mathrm{CI})$ & $\%$ & OR $(95 \% \mathrm{CI})$ & $\%$ & OR $(95 \% \mathrm{CI})$ \\
\hline Bullying behaviours ${ }^{\mathrm{b}}$ & 15.3 & Ref. & 18.5 & $\begin{array}{l}1.68 \\
(1.30-2.08)^{* *}\end{array}$ & 17.3 & $\begin{array}{l}1.14 \\
(0.98-1.29)\end{array}$ & 23.7 & $\begin{array}{l}1.62 \\
(1.39-1.85)^{* *}\end{array}$ & 16.6 & $1.10(0.97-1.22)$ \\
\hline Physical fights ${ }^{b}$ & 31.6 & Ref. & 50.2 & $1.21(0.86-1.45)$ & 30.9 & $\begin{array}{l}1.06 \\
(0.94-1.18)\end{array}$ & 37.9 & $\begin{array}{l}1.41 \\
(1.25-1.57)^{* *}\end{array}$ & 37.0 & $\begin{array}{l}1.23 \\
(1.12-1.34)^{* *}\end{array}$ \\
\hline
\end{tabular}

OR odds ratio, $C I$ confidence interval, FAS family affluence scale

${ }^{a}$ All OR were mutually adjusted for age, sex, and socioeconomic status as measured by the FAS

${ }^{b}$ Health complaints (range 0-32); low life satisfaction (Cantril scale $<6$ ); bulling others at school and physical fight (at least once)

$* * p<0.05$

discusses how involuntary immigration, driven by war or heavy political or economic strains, is an important source of stress that could negatively affect mental health (Aneshensel 1992). Furthermore, according to the acculturation theory, when immigrant youths acquire the host country's culture faster than their parents, it can foster a socalled acculturation gap, which could further increase the occurrence of mental stress in these youths (Cohen and Wills 1985). This situation, together with other immigration-related stressors, can set the stage for generational conflict and increase the risk of marginalisation (Berry 1997).

Our results on externalising behaviours showed that the risk of reporting bullying behaviours and physical fights were overrepresented in the first generation and decreased in the second generation of immigrants, independently of ethnic background. This reduction may be read as sign of sociocultural adaptation, in accordance with Argyle's theory, which describes this adaptation as a systemic process of learning the appropriate skills needed to operate effectively in a specific social milieu (Berry and Sam 1997).

Overall, the profiles that emerge from our results are coherent with those reported in Kouider's review (Kouider et al. 2014), but are different from those reported in other studies (Mood et al. 2016; Stevens et al. 2015). Unlike the conclusions reported by Stevens and colleagues (Stevens et al. 2015), we underlined a disadvantage in the second generation of immigrants, except in those coming from
Western countries. This difference seems to depend mainly on how subgroups of immigrants are defined and compared. In our study, the EU-15 subgroup was included in the Western countries group, and the EU-13 subgroup was combined with the non-Western/non-European group, since they showed similar SES characteristics and perceived well-being. On the contrary, Stevens et al. combined the EU-13 subgroup with the EU-15 subgroup, thus, in the authors' opinion, nullifying the second-generation effect.

Mood et al. (2016) suggested that the welfare policies of different hosting countries could have an effect on immigrant integration, and consequently on well-being. Thus, another explanatory factor for our results may be Italy's welfare policies regarding immigrants. The Migrant Integration Policy Index (MIPEX), a unique tool which measures the existence and impact of policies aimed at immigrant integration in the EU (Huddleston et al. 2015), placed Italy in the top 50\% when it comes to policies that are favourable to immigrant integration, giving a ranking of 13th place among the 38 EU Member States. However, this ranking descends to 23rd place in the area of education, where adolescents, and specifically second-generation adolescent immigrants, may be most affected (Huddleston et al. 2015).

In summary, according to the social stress and the acculturation theory, our results suggest that immigrants' ethnic background is a good predictor of their perceived well-being; however, that well-being also seems to depend 
on the immigrant policies that exist in the host country (Crul and Schneider 2010; Mood et al. 2016). Indeed, MIPEX results suggest that Italy should increase its investment in early prevention initiatives in schools. For example, by providing support to youths living in disadvantaged social or familial circumstances, providing support in intercultural education, and giving the proper training and professional support to school teachers and staff (Centers for Disease Control and Prevention 2009).

The results of this study should be considered in light of its limitations and strengths. First and foremost, this is a cross-sectional study, and therefore, it not allows us to draw any conclusions about causation. Moreover, part of the observed difference between the adolescents from the host country and their immigrant counterparts may reflect a systematic reporting difference attributable to the relationship between the culture of the respondent and the possible related, inherent biases. Such reporting differences have been documented in several cultural groups (Johnson et al. 2005), but Allen et al. (2016) found the validity of self-rated health to be similar for adolescents of different ethnic groups. Moreover, a high cross-cultural validity of self-reported externalising behaviours was also reported in the review by Paalman et al. (2013). As such, the difference in perceived well-being reported in our results is plausible. Furthermore, the dichotomisation of some of the variables under study (life satisfaction, bulling behaviours, and physical fights) could have led to a loss of information. However, we decided to follow faithfully the HBSC protocol recommendations (Currie et al. 2014) in order to allow comparability with 2016 results (Inchley et al. 2016) and with other published studies. Our results are limited to Italy (only one of the HBSC participating countries) and to a restricted age range (11-15 years old), thus limiting the generalisability of our findings. Finally, due to the study characteristics, it was not possible to distinguish or stratify on the different reasons for immigration (i.e. economic, political immigration, and refugee) or for length of stay.

Among the main strengths, this study is one of the first, to the authors' knowledge, to take into consideration immigrant status and adolescent's perceived well-being, while also taking into account sex, age, ethnic background, and being a first- or second-generation immigrant. Additionally, the study is based on a robust survey, the HBSC study, and the 2013/2014 wave of this study in Italy contains the largest sample size available in these developmental ages (Dalmasso et al. 2017). As such, it can positively contribute to the expanding research in this field.

Acknowledgements HBSC is a World Health Organisation crossnational study. The international coordinator for the 2014/2015 study was Johanna Inchley, University of St Andrews; Data Bank Manager: Oddrun Samdal, University of Bergen. In Italy, the study has been carried out since 2001 under the coordination of the Universities of
Torino, Padova, and Siena on the behalf of the Ministry of Education and the Ministry of Health.

Authors' contribution $\mathrm{AB}$ and PL edited the draft and completed the manuscript; both made the greatest contribution to the paper; LC and PD conducted the statistical analyses; PB, GL, AV contributed to the final manuscript editing. All authors participated in designing the study and data collection as members of the HBSC Italian team. All authors have critically revised the manuscript and approved its final version.

Funding The survey was partly funded by the Italian Ministry of Health (Grant No. cap.4393/2005-CCM).

\section{Compliance with ethical standards}

Conflict of interest All authors declare that they have no conflict of interest.

Ethical approval All procedures performed in this study were in accordance with the ethical standards of the research committee recommendations and with the 1964 Helsinki Declaration and its later amendments.

Informed consent Informed consent was obtained from all individual participants included in the study at local authority, school, parent/guardian, and student levels. No reimbursement was planned nor provided for participation.

\section{References}

Allen CD, McNeely CA, Orme JG (2016) Self-rated health across race, ethnicity, and immigration status for US adolescents and young adults. J Adolesc Health 58(1):47-56

Aneshensel CS (1992) Social stress: theory and research. Ann Rev Sociol 18(1):15-38

Berry JW (1997) Immigration, acculturation, and adaptation. Appl Psychol 46(1):05-34. https://doi.org/10.1111/j.1464-0597.1997. tb01087.x

Berry JW, Sabatier C (2011) Variations in the assessment of acculturation attitudes: their relationships with psychological wellbeing. Int J Intercult Relat 35(5):658-669

Berry JW, Sam DL (1997) Acculturation and adaptation. In: Berry JW, Poortinga YH, Pandey J (eds) Handbook of cross-cultural psychology: social behavior and applications, vol 3. Allyn \& Bacon Needham Heights, MA, pp 291-326

Cantril H (1965) The pattern of human concerns. Rutgers University Press, New Brunswick

Centers for Disease Control and Prevention (2009) School connectedness: strategies for increasing protective factors among youth. US Department of Health and Human Services, Atlanta

Cohen S, Wills TA (1985) Stress, social support, and the buffering hypothesis. Psychol Bull 98(2):310

Crul M, Schneider J (2010) Comparative integration context theory: participation and belonging in new diverse European cities. Ethn Racial Stud 33(7):1249-1268

Currie C, Griebler R, Inchley J et al (2010) Health Behaviour in School-aged Children (HBSC) study protocol: background, methodology and mandatory items for the 2009/10 survey. CAHRU, Edinburgh

Currie C, Inchley J, Molcho M, Lenzi M, Veselska Z, Wild F (eds) (2014) Health Behaviour in School-aged Children (HBSC) study 
protocol: background, methodology and mandatory items for the 2013/14 survey. CAHRU, St Andrews

Dalmasso P, Borraccino A, Lazzeri G et al (2017) Being a young migrant in Italy: the effect of perceived social support in adolescence. J Immigr Minor Health 1-18 (in press)

Djerboua M, Chen BE, Davison CM (2016) Physical fighting, fighting-related injuries and family affluence among Canadian youth. BMC Public Health 16(1):199

Dodge R, Daly AP, Huyton J, Sanders LD (2012) The challenge of defining wellbeing. Int J Wellbeing 2(3):222-235

Eurostat (2018) Eurostat: statistic explained, EU enlargements. In. http://ec.europa.eu/eurostat/statistics-explained/index.php/Glos sary:EU_enlargements. Accessed 4 Sept 2018

Harker K (2001) Immigrant generation, assimilation, and adolescent psychological well-being. Soc Forces 79(3):969-1004

Haugland S, Wold B (2001) Subjective health complaints in adolescence-reliability and validity of survey methods. J Adolesc 24(5):611-624

Hernandez DJ (2010) Internationally comparable indicators for children of immigrants. Child Indic Res 3(4):409-411

Huddleston T, Bilgili O, Joki A-L, Vankova Z (2015) The Migrant Integration Policy Index (MIPEX). In: CIDOB. http://www. mipex.eu/. Accessed 27 July 2018

Inchley J, Currie D, Taryn Y et al (eds) (2016) Growing up unequal: gender and socioeconomic differences in young people's health and well-being. World Health Organization, Copenaghen

ISTAT (2016) Migrazioni internazionali e interne della popolazione residente 2015. In: Statistiche ISTAT Report (ed). Roma

Johnson T, Kulesa P, Cho YI, Shavitt S (2005) The relation between culture and response styles: evidence from 19 countries. J Cross Cult Psychol 36(2):264-277

Kim KJ, Conger RD, Elder GH Jr, Lorenz FO (2003) Reciprocal influences between stressful life events and adolescent internalizing and externalizing problems. Child Dev 74(1):127-143

Kouider EB, Koglin U, Petermann F (2014) Emotional and behavioral problems in migrant children and adolescents in Europe: a systematic review. Eur Child Adolesc Psychiatry 23(6):373-391

Kouider EB, Koglin U, Petermann F (2015) Emotional and behavioral problems in migrant children and adolescents in American countries: a systematic review. J Immigr Minor Health 17(4):1240-1258

Kwak K (2016) An evaluation of the healthy immigrant effect with adolescents in Canada: examinations of gender and length of residence. Soc Sci Med 157:87-95

MIUR (2015) Gli alunni stranieri nel sistema scolastico italiano: A.S. 2014/2015. MIUR - Ufficio di Statistica, Roma

Mood C, Jonsson JO, Låftman SB (2016) Immigrant integration and youth mental health in four European countries. Eur Sociol Rev 32(6):716-729

Paalman CH, Terwee CB, Jansma EP, Jansen LM (2013) Instruments measuring externalizing mental health problems in immigrant ethnic minority youths: a systematic review of measurement properties. PLoS One 8(5):e63109

Pickett W, Iannotti RJ, Simons-Morton B, Dostaler S (2009) Social environments and physical aggression among 21,107 students in the United States and Canada. J Sch Health 79(4):160-168

Ravens-Sieberer UKG, Thomas C (2004) School and health. In: Currie C (ed) Young people's health in context health policy for children and adolescents, vol 4. WHO Regional Office for Europe, Copenhagen

Resnick MD, Bearman PS, Blum RW et al (1997) Protecting adolescents from harm: findings from the National Longitudinal Study on Adolescent Health. JAMA 278(10):823-832

Sam DL, Vedder P, Liebkind K, Neto F, Virta E (2008) Immigration, acculturation and the paradox of adaptation in Europe. Eur J Dev Psychol 5(2):138-158

Stefanek E, Strohmeier D, Fandrem H, Spiel C (2012) Depressive symptoms in native and immigrant adolescents: the role of critical life events and daily hassles. Anxiety Stress Coping 25(2):201-217

Stevens GW, Walsh SD, Huijts T et al (2015) An internationally comparative study of immigration and adolescent emotional and behavioral problems: effects of generation and gender. J Adolesc Health 57(6):587-594

Torsheim T, Cavallo F, Levin KA et al (2016) Psychometric validation of the revised family affluence scale: a latent variable approach. Child Indic Res 9(3):771-784 